\title{
QAGUPUBLICATIONS
}

[Global Biogeochemical Cycles]

Supporting Information for

\section{Controls on Dissolved Silicon Isotopes along the US GEOTRACES Eastern Pacific Zonal Transect (GP16)}

\author{
[P. Grasse ${ }^{1,2}$, I. Closset ${ }^{3}$, J. L. Jones ${ }^{3}$, S. Geilert ${ }^{2}$, and M.A. Brzezinski ${ }^{3}$ ]
}

${ }^{1}$ German Centre for integrative Biodiversity Research (iDiv)

Deutscher Platz 5E, 04103 Leipzig

Germany

${ }^{2}$ GEOMAR Helmholtz Centre for Ocean Research Kiel

Ocean Circulation and Climate Dynamics

Wischhofstr. 1-3

24148 Kiel,

Germany

${ }^{3}$ Marine Science Institute and the Department of Ecology, Evolution, and Marine

Biology

University of California,

Santa Barbara, CA 93106

USA

\section{Contents of this file}

Figures $\mathrm{S} 1$ to $\mathrm{S} 6$

Tables S1 to S3 


\section{Additional Supporting Information}
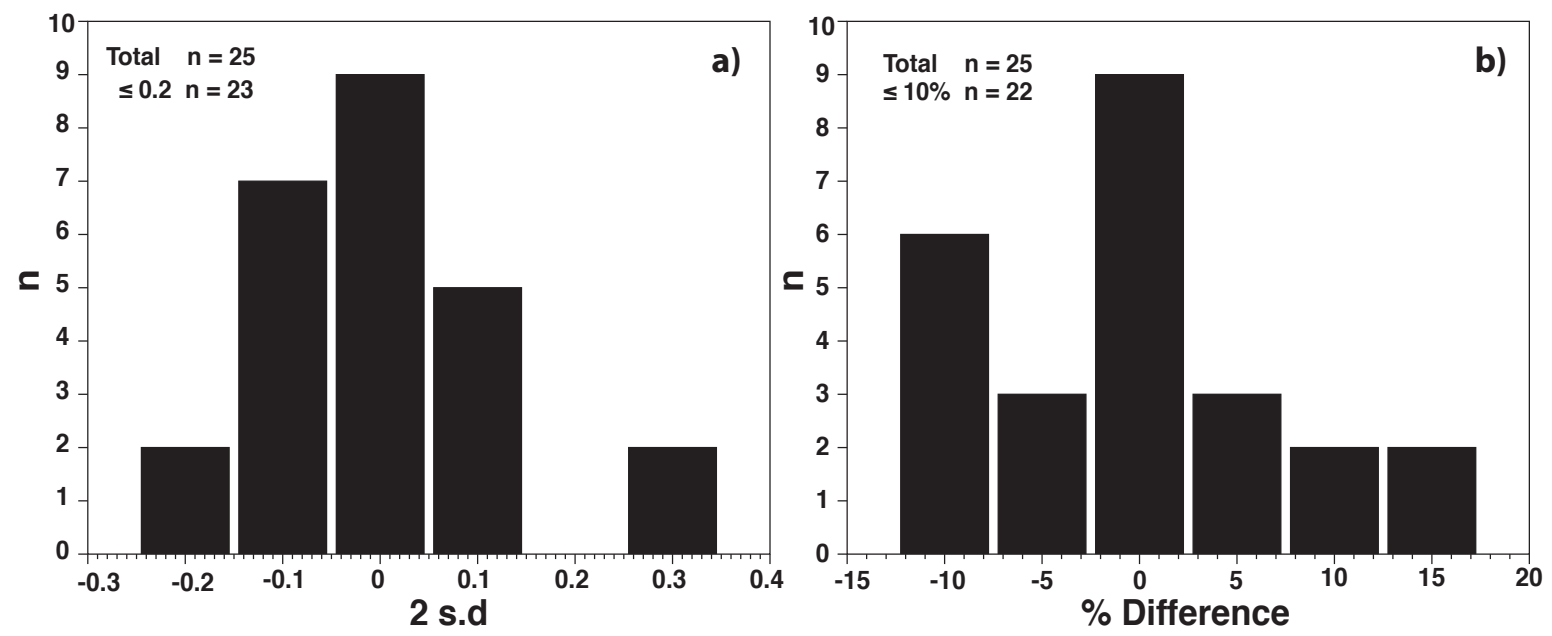

Figure S1. (a) Number of samples (n) with their respective external reproducibility (2 s.d.) and (b) $\%$ difference between $\delta^{30} \mathrm{Si}$ measurements from GEOMAR and UCSB. See also Table S2 and S3.

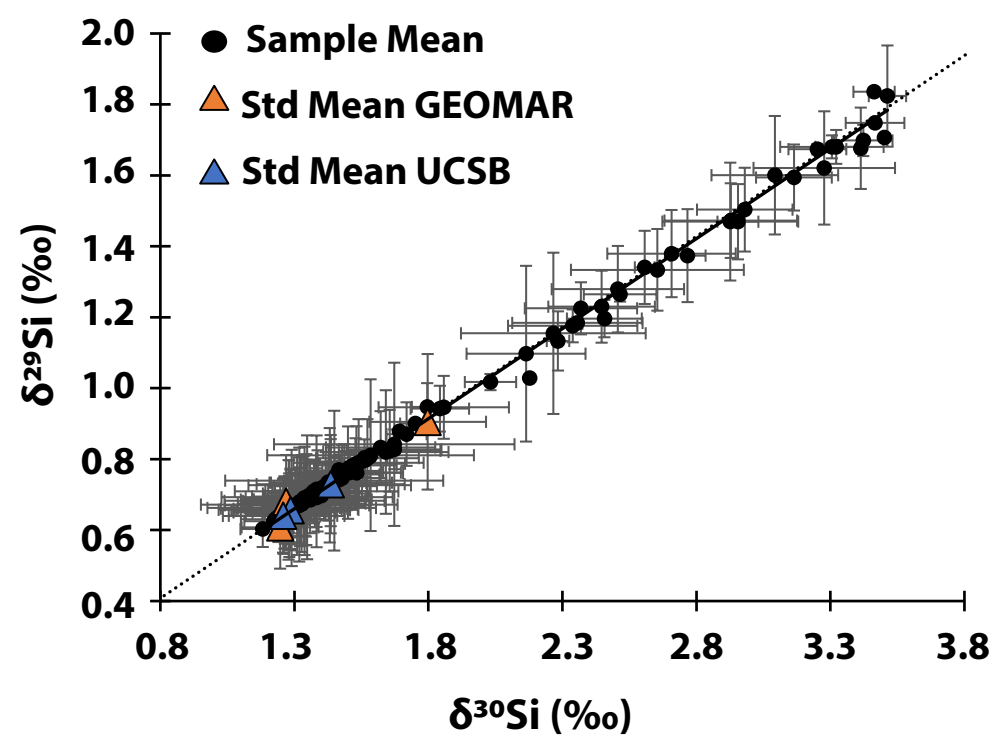

Figure S2. $\delta^{30} \mathrm{Si}$ vs $\delta^{29} \mathrm{Si}$ of sample means (black dots), means of reference materials analyzed at GEOMAR (orange triangles) and the means of reference materials analyzed at UCSB (blue triangles). Error bars represent the average standard deviation $( \pm 0.2 \%$ ). The black line is the least square linear regression between $\delta^{30} \mathrm{Si}$ and $\delta^{29} \mathrm{Si}$ of the sample datasets $\left(\delta^{30} \mathrm{Si}=0.5078 \times \delta^{29} \mathrm{Si}, \mathrm{R}^{2}=0.9972\right)$, and the dotted line represents the theoretical kinetic fractionation law $\left(\delta^{30} \mathrm{Si}=0.5092 \times \delta^{29} \mathrm{Si}\right.$, as calculated following Young et al. (2002). Note that, for clarity, Big Batch values are not shown on the plot but fall on the same fractionation line (see Table. S3). 


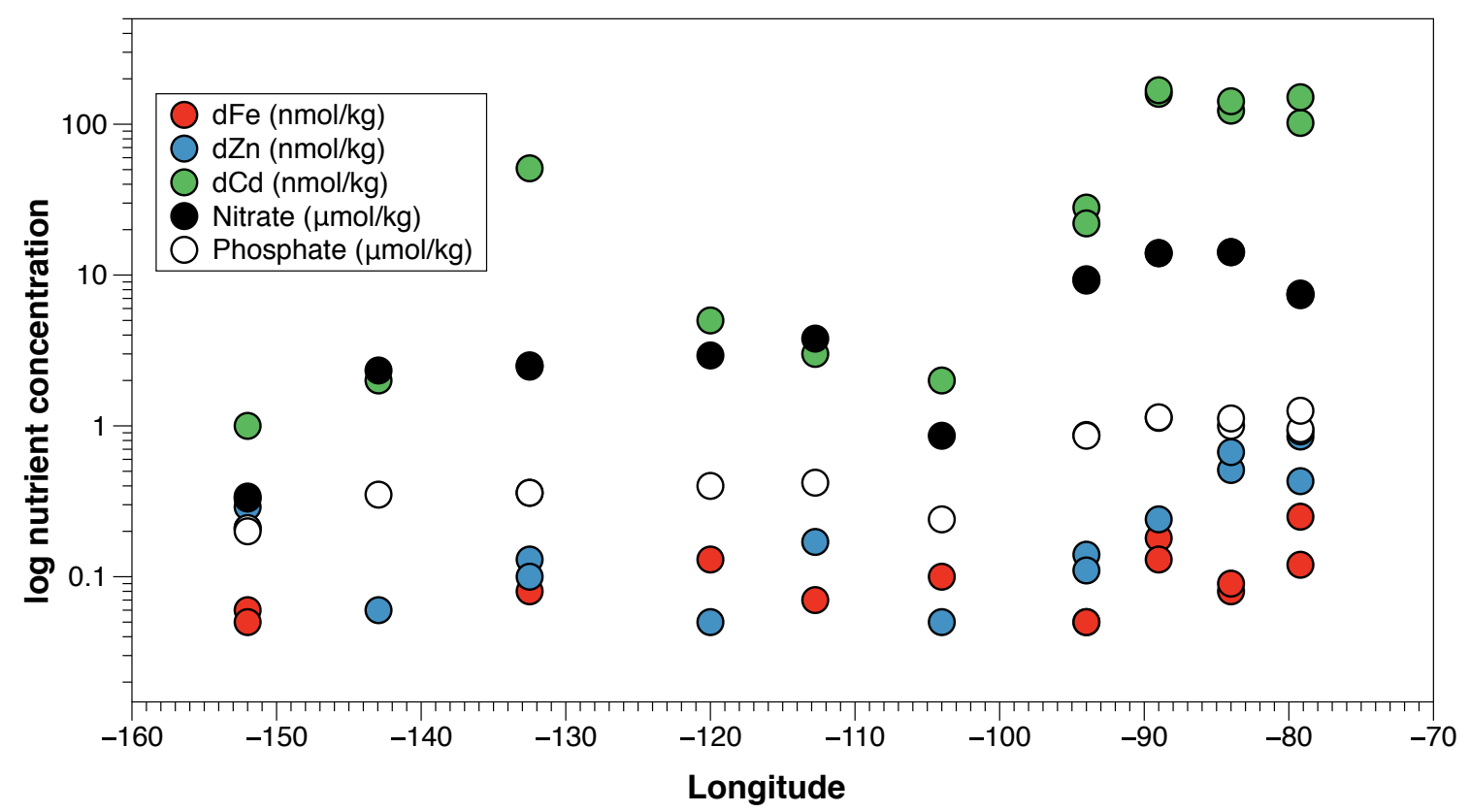

Figure S3. Concentrations of macronutrients $\left(\left[\mathrm{PO}_{4}{ }^{3-}\right],\left[\mathrm{NO}_{3}{ }^{-}\right]\right)$as well as micronutrients (dissolved iron (dFE), zinc (dZn), cadmium(dCd)) in the upper $50 \mathrm{~m}$ across the transect. Please note the logarithmic scale for nutrient concentrations as well as different units: $\left[\mathrm{PO}_{4}{ }^{3-}\right],\left[\mathrm{NO}_{3}^{-}\right]$) concentrations are in $\mu \mathrm{mol} \mathrm{kg}{ }^{-1}$ and $\mathrm{dFe}, \mathrm{dZn}, \mathrm{dCd}$ in $\mathrm{nmol} \mathrm{kg}{ }^{-1}$. Trace metal data is adapted from John et al. $(2017 ; 2018)$. 

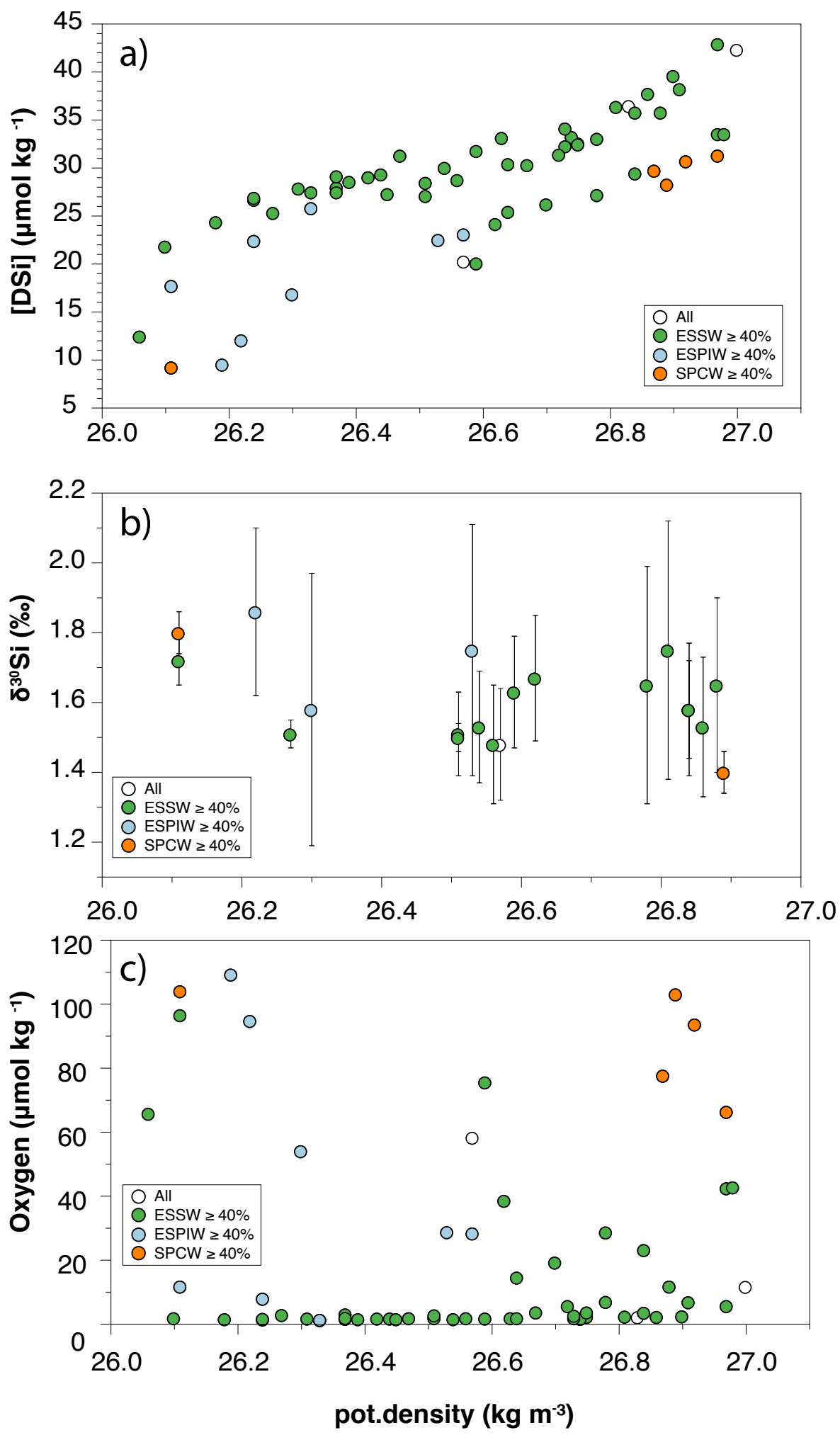

Figure S4. (a) Distribution of [DSi], (b) $\delta^{30} \mathrm{Si}$ and (c) oxygen concentrations in subsurface waters (potential density: 26 to $27 \mathrm{~kg} / \mathrm{m}^{3}$ ). Samples with ESSW, ESPIW and SPCW water mass contribution over $40 \%$ are marked in green, blue and orange, respectively. 


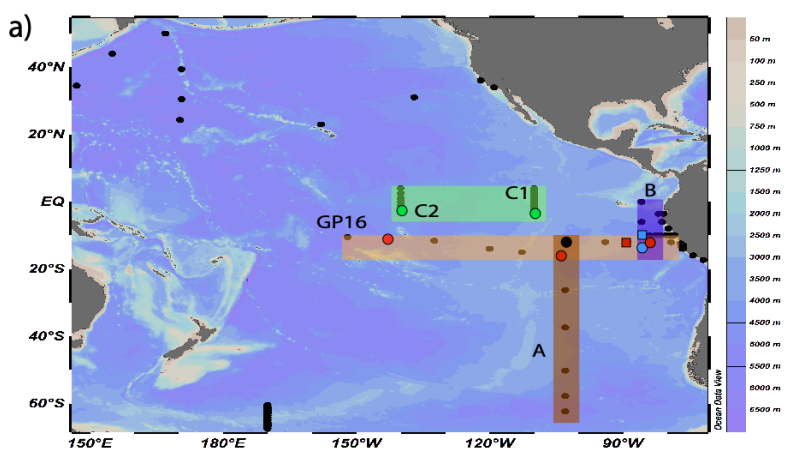

- GP16 (This study)

- de Souza et al., 2012a (A)

- Grasse et al., 2013 (B)

Beucher et al., 2008(C1); 2011 (C2)
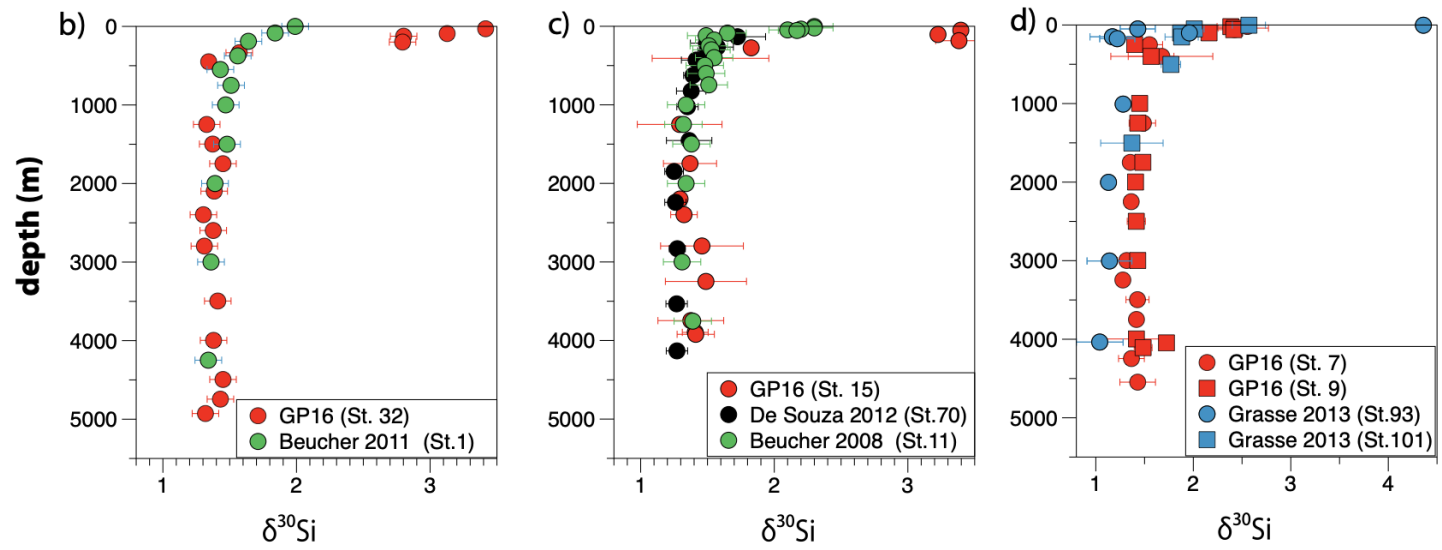

Figure S5. (a) Map showing sampling locations for $\delta^{30} \mathrm{Si}$ in the Pacific with four highlighted transects in the Eastern Pacific. The GP 16 transect is marked in red. The data by de Souza et al. (2012a; A) in grey. Data from offshore transects by Grasse et al. (2013, B) in blue and Beucher et al. (2008, C1) and Beucher et al. (2011, C2) in green. (b) Comparison between Beucher et al. (2011, St. 1) and GP16 (St. 32) (c). Comparison between data from de Souza et al. (2012a, St. 70), GP 16 (Sts. 15) and Beucher et al. (2008, St. 11) (d) Comparison between data from Grasse et al. (2013; Sts.93, 101) and GP16 (Sts. 7, 9). Individual error bars indicate the reproducibility of analytical replicates ( 2 s.d.) as given in the references. Please note the different scale for (d). 

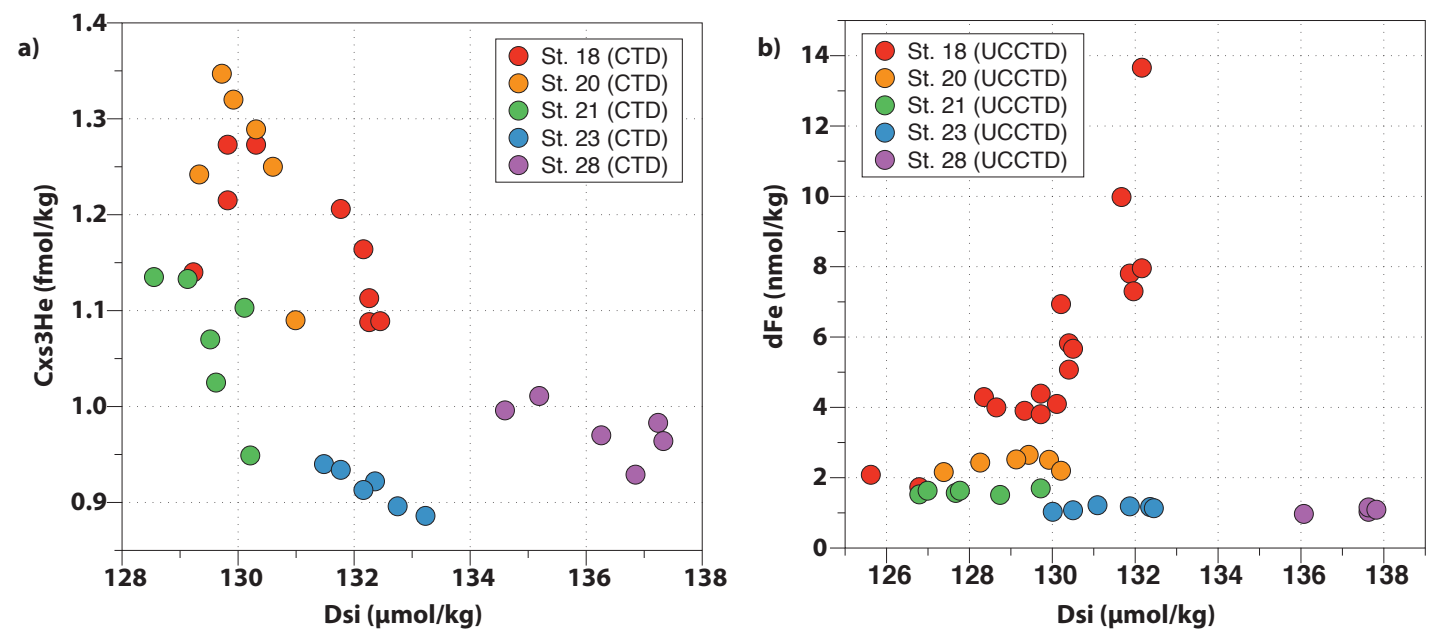

Figure S6: (a) Excess ${ }^{3} \mathrm{He}\left(\mathrm{C}_{\mathrm{ex}}{ }^{3} \mathrm{He}\right)$ in fmol kg-1 (data adapted from Jenkins et al., 2018) versus DSi (in $\left.\mu \mathrm{mol} \mathrm{kg}{ }^{-1}\right)$, (b) DSi $\left(\mu \mathrm{mol} \mathrm{kg}{ }^{-1}\right)$ versus $\mathrm{dFe}\left(\mathrm{nmol} \mathrm{kg}^{-1}\right)$ above and westward of the EPR (Sts. 18, 20, 23, 28). Samples during GP16 were taken with a conventional CTD (CTD; Helium samples) and with an ultra-clean CTD (UCCTD, dFe samples). [DSi] measured in CTD and UCCTD samples showed small differences (< $5 \%$ ). Therefore, we plotted Helium versus [DSi] from CTD samples and [dFe] versus DSi from UCCTD samples. 
Table S1. Sampling locations with $\delta^{30} \mathrm{Si}$ measurements and nutrient concentrations ([Si], $\left[\mathrm{NO}_{3}^{-}\right]$, [ $\left.\mathrm{PO}_{4}^{-}\right]$) as well as hydrography data. The external reproducibility (2 s.d.) for $n$ analytical replicates is given. In case samples were measured at both laboratories (GEOMAR and UCSB) 2 s.d represents the external reproducibility of all single measurements. Further details on the inter-calibration between both laboratories are in the supplementary (Tabs. S1, S2). $\delta^{30} \mathrm{Si}$ data is available through the Biological and Chemical Oceanography Data Management Office Website (https://www.bco-dmo.org/dataset/728819).

\begin{tabular}{|c|c|c|c|c|c|c|c|c|c|c|c|c|c|c|c|c|c|c|}
\hline Station & $\begin{array}{l}\text { GEOTRACES } \\
\text { SAMPLE Nr. }\end{array}$ & Lat & Long & $\begin{array}{l}\text { Bottom Depth } \\
\text { (m) }\end{array}$ & $\begin{array}{l}\text { DEPTH } \\
(\mathrm{m})\end{array}$ & $\begin{array}{l}\text { Temp } \\
\left({ }^{\circ} \mathrm{C}\right)\end{array}$ & Salinity & $\begin{array}{c}\text { Pot. } \\
\text { Density } \\
(\mathrm{kg} / \mathrm{m} 3)\end{array}$ & $\begin{array}{c}\text { Oxygen } \\
(\mu \mathrm{mol} / \mathrm{kg})\end{array}$ & $\begin{array}{c}\text { DSi } \\
(\mu \mathrm{mol} / \mathrm{kg})\end{array}$ & $\begin{array}{c}\text { Nitrate } \\
\text { (umol/kg) }\end{array}$ & $\begin{array}{l}\text { Phosphate } \\
\text { (umol/kg) }\end{array}$ & $\begin{array}{c}\text { Dsi } / \mathrm{N} \\
\text { (umol/kg) }\end{array}$ & $\begin{array}{c}\delta^{30} \mathrm{Si} \\
(\%)\end{array}$ & 2 s.d. & Lab & $\ln (D s i)$ & $\mathrm{si}^{*}$ \\
\hline 1 & 2143 & -12.01 & -79.20 & 5526 & 19.7 & 17.63 & 35.19 & 25.51 & 241.7 & 2.54 & 7.43 & 0.92 & 0.34 & 2.95 & 0.08 & 2 GEOMAR & 0.93 & -4.89 \\
\hline 1 & 2142 & -12.01 & -79.20 & 5526 & 28.6 & 17.61 & 35.19 & 25.52 & 239.9 & 2.44 & 7.54 & 0.95 & 0.32 & 2.93 & 0.25 & 3 GEOMAR & 0.89 & -5.1 \\
\hline 1 & 2140 & -12.01 & -79.20 & 5526 & 64.3 & 16.60 & 35.12 & 25.70 & 187.9 & 5.46 & 11.38 & 1.52 & 0.48 & 2.28 & 0.04 & 3 GEOMAR & 1.70 & -5.92 \\
\hline 1 & 38 & -12.01 & -79.20 & 5526 & 130.2 & 13.38 & 34.94 & 26.27 & & 25.37 & 22.22 & 2.43 & 1.14 & 1.51 & 0.042 & 2/1 GEOMAR/UCSB & 3.23 & 3.15 \\
\hline 1 & & -12.01 & -79.20 & 26 & 261.1 & 11.82 & 34.85 & 26.51 & 2 & 28.5 & 27.93 & 2.54 & 1.02 & 1.51 & 0.12 & 2 GEOMAR & & 0.57 \\
\hline 1 & 2081 & -12.01 & -79.20 & 25 & 449.9 & 8.97 & 34.66 & 26.86 & 2.4 & 37.77 & 37.88 & 2.88 & 1.00 & 1.53 & 0.203 & 3/3 GEOMAR/UCSB & 63 & -0.11 \\
\hline 1 & 2078 & -12 & -79.20 & 5525 & 799.6 & 5.51 & 34.54 & 27.25 & 24.4 & 69.02 & 45.52 & 3.24 & 1.52 & 1.51 & & 1 UCSB & 23 & 23.5 \\
\hline 1 & 76 & -12 & -79.20 & 5525 & 1201.5 & 3.82 & 34.57 & 27.46 & 64.9 & 102.11 & 42.5 & 3.1 & 2.40 & 1.36 & 0.182 & 2/2 GEOM & 4.63 & 59.61 \\
\hline 1 & & -12 & -79.20 & 25 & 2002.4 & 2.29 & 34.64 & 27.66 & 101.1 & 42.9 & 39.31 & 2.89 & 64 & 1.31 & 0.27 & & 4.96 & 103.59 \\
\hline 1 & & -12.01 & -79.20 & 25 & 2400.9 & 1.95 & 34.66 & 27.71 & 121.9 & 143.58 & 37.7 & 2.75 & 81 & 1.30 & 0.11 & 5 UCSB & 4.97 & 105.88 \\
\hline 1 & 2072 & -12.01 & -79.20 & 25 & 2800.7 & 1.80 & 34.68 & 27.73 & 129.2 & 148.56 & 37.3 & 2.71 & 3.98 & 1.43 & 0.09 & 4 UCSB & 5.00 & 111.26 \\
\hline 1 & 71 & -12.01 & -79.20 & 25 & 3198.1 & 1.80 & 34.68 & 27.73 & 130.8 & 150.61 & 37.2 & 2.7 & 4.05 & 1.31 & 0.08 & 7 UCSB & 5.01 & 113.41 \\
\hline 1 & 2251 & -12.01 & -79.20 & 27 & 3194.8 & 1.79 & 34.68 & 27.73 & 131.1 & 149.83 & 37.32 & 2.7 & 4.01 & $1.31 \sim$ & & 1 UCSB & 5.01 & 112.51 \\
\hline 1 & 2250 & -12.01 & -79.20 & 5527 & 3594.9 & 1.80 & 34.68 & 27.73 & 134.4 & 148.75 & 36.99 & 2.68 & 4.02 & 1.34 & \begin{tabular}{|l|}
0.10 \\
\end{tabular} & 3 UCSB & 5.00 & 111.76 \\
\hline 1 & 2248 & -12.01 & -79.20 & 527 & 4393.5 & \begin{tabular}{|l|l}
1.79 \\
\end{tabular} & 34.69 & 27.74 & 52.2 & 135.28 & 35.56 & 2.58 & 3.80 & 1.35 & 0.11 & 2 UCSB & 4.91 & \begin{tabular}{|l}
99.72 \\
\end{tabular} \\
\hline 1 & 2245 & -12.01 & -79.20 & 27 & 5040.8 & 1.83 & 34.69 & 27.74 & & & 34.89 & & 3.80 & 1.35 & 0.13 & 4 UCSB & 4.89 & 97.76 \\
\hline 1 & 2243 & -12 & -79 & & 5317 & 1.85 & 34.69 & 27.74 & & & & & 77 & $1.30 \sim$ & & 1 UCSB & 4.88 & 96.62 \\
\hline 1 & 2240 & -12.01 & -79.20 & 27 & 5500.3 & 1.88 & 34.69 & 27.73 & 158.3 & 131.48 & & 2.57 & 3.79 & 1.29 & 0.18 & 4 UCSB & 4.88 & 96.8 \\
\hline 7 & & -12.00 & -84.00 & 4553 & 19.5 & 18.95 & 35.29 & 25.26 & 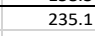 & 4.68 & & 1 & 33 & 2.46 & $\begin{array}{ll}0.14 \\
\end{array}$ & 2 GEOM & 54 & -9.51 \\
\hline 7 & & -12. & & & & & & & & & & 2 & 34 & 2.34 & .4 & 3 GEO & 0 & -9.4 \\
\hline 7 & & -12.00 & -84.00 & & 58.9 & & & & 220.6 & 56 & & 1.4 & 38 & 2.37 & .21 & $3 \mathrm{GEO}$ & 72 & -9.26 \\
\hline 7 & 167 & -12.00 & -84.00 & 553 & 251 & 11.56 & 34.83 & 26.54 & 1.7 & 30.06 & 23.82 & 2.58 & 1.26 & 1.53 & 0.162 & 2/2 GEOMAR/UCSB & 3.40 & 6.24 \\
\hline 7 & 3155 & -12.00 & -84.00 & 46 & 399.1 & 9.39 & 34.69 & 26.81 & 2.5 & 36.41 & 36.53 & 2.8 & 1.00 & 1.75 & 0.372 & 2/4 GEOM & 3.59 & -0.12 \\
\hline 7 & 3149 & -12.00 & -84.00 & 16 & 1249.4 & 3.58 & 34.57 & 27.49 & 70.2 & 107.08 & 42. & 3.11 & 2.52 & 1.42 & 0.272 & 2/5 GEOMA & 4.67 & 64.56 \\
\hline 7 & 3147 & -12.00 & -84.00 & 4546 & 1749.1 & 2.51 & 34.63 & 27.63 & 95.9 & 135.58 & 40. & 2.99 & 3.37 & $1.37 \sim$ & & 1 UCSB & 4.91 & 95.34 \\
\hline 7 & 3145 & -12.00 & -84.00 & 4546 & 2249.2 & 2.05 & 34.66 & 27.69 & 113.6 & 146.31 & 38.79 & 2.86 & 3.77 & 1.43 & 0.21 & 2 UCSB & 4.99 & 107.52 \\
\hline & 3264 & -12.00 & -84.00 & 61 & 2996 & 1.80 & 34.68 & 27.73 & 131.7 & 149.92 & 37.23 & 2. & 4.03 & 1.27 & 0.13 & 2 UCSB & 5.01 & 112.69 \\
\hline 7 & 3263 & -12.00 & -84.00 & 4561 & 3244.4 & 1.79 & 34.68 & 27.7 & 136.9 & & & 2. & 4.00 & 1.18 & 0.08 & 3 UCSB & 4.99 & 110.3 \\
\hline 7 & 3262 & $-12 . c$ & & 4561 & 3494.3 & 1.78 & 34.69 & 27.7 & 144.7 & 140.55 & 3 & 2.64 & 89 & 1.38 & 0.17 & 4 UCSB & 4.95 & 104.42 \\
\hline & 2 & -12 & & 4561 & 3746.1 & 1.77 & 34.69 & 2 & 148.3 & 72 & & 262 & 4 & 1.39 & 0.19 & 3 UCSB & 3 & 101.83 \\
\hline & & -12 & & 4561 & 42 & 1.80 & 34 & 2 & & 13 & & 2.6 & & 1.38 & 0.12 & 2 UCSB & 2 & 100.84 \\
\hline & 32 & -12 & -8 & 4561 & 4543.2 & $\begin{array}{l}1.80 \\
1.84 \\
\end{array}$ & 34.69 & 27 & 150.2 & $\begin{array}{l}130.65 \\
\end{array}$ & 35.64 & 2. & 3 & 1.28 & 0.21 & 4 UCSB & 2 & 101.01 \\
\hline 9 & 340 & -12 . & -89 & 4137 & 20.6 & 19.07 & 35.36 & 25 & 23 & 5.37 & 14 & 1.13 & 38 & 2.31 & 0.312 & $2 / 1$ GEOMAR/UCSB & 68 & -8.63 \\
\hline 9 & 340 & -12 & -89 & 4137 & 39.8 & 19.03 & 35.36 & 25 & 23 & 5.37 & 3.94 & 1.14 & & 2.52 & 0.14 & $2 \mathrm{GEC}$ & & -8.57 \\
\hline 9 & & -12 & -89 & & 59.4 & 18.17 & 35 & 25 & 22 & 4.59 & & 1. & & 2.36 & 0.24 & $2 \mathrm{GEC}$ & 52 & -7.87 \\
\hline 9 & 34 & -12 & & & 99.4 & 16.08 & & & 124.5 & 1 & & & & 2.03 & 0.10 & 3 GEON & .09 & -10.82 \\
\hline 9 & 3394 & -12.00 & -89. & 41 & 248.1 & 11.41 & 34. & 26 & 2 & 28.79 & & 2. & 0 & 1.48 & 0.172 & 2/3 GEOMAR/UCSB & 3.50 & 0.03 \\
\hline 9 & 3346 & -12.00 & -89 & 41 & 397.7 & 9.12 & 34.67 & 26 & 3.7 & 35 & 37 & 2.82 & 0.95 & 1.58 & 0.191 & 1/3 GEOMAR/UCS & 3.58 & -1.94 \\
\hline 9 & 3341 & -12.00 & -89 & 4130 & 993.5 & 4.45 & 34.54 & 27 & 56.7 & 83 & 43 & 3.13 & 1.92 & $1.51 \sim$ & & 1 UCSB & 4.42 & 39.92 \\
\hline 9 & 3340 & -12.00 & -89.00 & 4130 & 1243.1 & 3.56 & 34.57 & 27.49 & 75.3 & 103.86 & 41. & 3.07 & 8 & 1.47 & 0.142 & 2/3 GEOMAR/UCSB & 4.64 & 62 \\
\hline 9 & 3338 & -12.00 & -89.00 & 4130 & 1740.5 & 2.54 & 34.63 & 27.63 & 99.2 & 130.21 & 39. & 2.96 & 3.28 & 1.36 & 0.22 & 3 UCSB & 4.87 & 90.5 \\
\hline 9 & 3337 & -12.00 & -89.00 & 0 & 1989.3 & 2.27 & 34.64 & 27.66 & 106.2 & 138.8 & 39 & 1 & 6 & $1.35 \sim$ & & 1 UCSB & 93 & 99.77 \\
\hline 9 & 3453 & -12. & -89.00 & 4138 & 2485.1 & 1.88 & 34.67 & 27.72 & 12 & 143.78 & 37 & 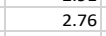 & 2 & 1.38 & 0.19 & 3 UCSB & 7 & 106.16 \\
\hline 9 & 3451 & -12.00 & -89.00 & 4138 & 2981.5 & 1.78 & 34.68 & 27.73 & 140. & 140.65 & 36.67 & 2.6 & 3.84 & 1.28 & 0.26 & 4 UCSB & 4.95 & 103.98 \\
\hline 9 & 344 & -120 & -89 & 017 & 2075 & 18 & & & & & & & 20 & & & 1 UCSB & & 119 \\
\hline 9 & 3443 & -12.00 & -89.00 & 4138 & 4085 & 1.83 & 34.69 & 27.73 & 145 & 38.11 & 36.29 & 2.63 & 3.81 & 1.45 & 0.01 & UCSB & 4.93 & 101.82 \\
\hline
\end{tabular}


Table S1. Continued

\begin{tabular}{|c|c|c|c|c|c|c|c|c|c|c|c|c|c|c|c|c|c|c|}
\hline Station & $\begin{array}{l}\text { GEOTRACES } \\
\text { SAMPLE Nr. }\end{array}$ & Lat & Long & $\begin{array}{c}\text { Bottom Depth } \\
(\mathrm{m})\end{array}$ & $\begin{array}{c}\text { DEPTH } \\
(\mathrm{m})\end{array}$ & $\begin{array}{c}\text { Temp } \\
\left({ }^{\circ} \mathrm{C}\right)\end{array}$ & Salinity & $\begin{array}{c}\text { Pot. } \\
\text { Density } \\
(\mathrm{kg} / \mathrm{m} 3) \\
\end{array}$ & $\begin{array}{c}\text { Oxygen } \\
(\mu \mathrm{mol} / \mathrm{kg})\end{array}$ & $\begin{array}{c}\mathrm{DSi} \\
(\mu \mathrm{mol} / \mathrm{kg})\end{array}$ & $\begin{array}{c}\text { Nitrate } \\
\text { (umol/kg) }\end{array}$ & $\begin{array}{l}\text { Phosphate } \\
\text { (umol/kg) }\end{array}$ & $\begin{array}{c}\begin{array}{c}D s i / N \\
\text { (umol/kg) }\end{array}\end{array}$ & $\begin{array}{l}\delta^{30} \mathrm{Si} \\
(\%)\end{array}$ & $\mathrm{n}$ & Lab & $\ln$ (Dsi) & $\mathrm{Si}^{*}$ \\
\hline 11 & 3578 & -12.00 & -94.00 & 3660 & 39.8 & 320.96 & 35.48 & 24.87 & 225.6 & 3.32 & 9.19 & 0.86 & 0.36 & 2.65 & 0.32 & 2 GEOMAR & 1.20 & -5.87 \\
\hline 11 & 3576 & -12.00 & -94.00 & 3660 & 110.9 & 16.90 & 35.14 & 25.65 & 161 & 3.61 & 11.62 & 1.13 & 0.31 & 2.16 & 0.22 & 2 GEOMAR & 1.28 & -8.01 \\
\hline 11 & 3575 & -12.00 & -94.00 & 3660 & 130.1 & 15.41 & 34.96 & 25.85 & 123.1 & 6.44 & 16.62 & 1.5 & 0.39 & 1.84 & 0.11 & 2 GEOMAR & 1.86 & -10.18 \\
\hline 11 & 3569 & -12.00 & -94.00 & 3660 & 259.4 & 411.45 & 34.76 & 26.51 & 2.9 & 27.13 & 26.54 & 2.65 & 1.02 & 1.50 & $0.041 /$ & 1/1 GEOMAR/UCSB & 3.30 & 0.59 \\
\hline 11 & 3529 & -12.00 & -94.00 & 3660 & 399.9 & 8.76 & 34.65 & 26.88 & 11.9 & 35.82 & 38.58 & 2.76 & 0.93 & 1.65 & $0.251 / 2$ & 1/2 GEOMAR/UCSB & 3.58 & -2.76 \\
\hline 11 & 3526 & -12.00 & -94.00 & 3660 & 699.3 & $\begin{array}{l}3 \quad 5.82 \\
\end{array}$ & 34.53 & 27.20 & 35.7 & 58.18 & 44.06 & 3.08 & 1.32 & 1.35 & 0.07 & 2 UCSB & 4.06 & 14.12 \\
\hline 11 & 3524 & -12.00 & -94.00 & 3660 & 899.3 & \begin{tabular}{|l|l|}
3 & 4.73 \\
\end{tabular} & 34.53 & 27.33 & 57.2 & 75.75 & 43.23 & 3.08 & 1.75 & 1.39 & 0.11 & 2 UCSB & 4.33 & 32.52 \\
\hline 11 & 3522 & -12.00 & -94.00 & 3660 & 1249.4 & $4 \quad 3.51$ & 34.57 & 27.49 & 79.9 & 104.84 & 41.39 & 3.05 & 2.53 & 1.43 & $0.092 /$ & 2/1 GEOMAR/UCSB & 4.65 & 63.45 \\
\hline 11 & 3520 & -12.00 & -94.00 & 3660 & 1751.1 & 2.56 & 34.62 & 27.62 & 105.1 & 128.36 & 39.31 & 2.92 & 3.27 & $1.37 \sim$ & & 1 UCSB & 4.85 & 89.05 \\
\hline 11 & 3698 & -12.00 & -94.00 & 3664 & 2247.3 & \begin{tabular}{|l|l|} 
& 1.99 \\
\end{tabular} & 34.66 & 27.70 & 125.3 & 141.43 & 37.66 & 2.74 & 3.76 & $1.30 \sim$ & & 1 UCSB & 4.95 & 103.77 \\
\hline 11 & 3697 & -12.00 & -94.00 & 3664 & 2497.1 & $\begin{array}{ll}1 & 1.83 \\
\end{array}$ & 34.67 & 27.72 & 2.7 & 136.75 & 36.29 & 2.63 & 3.77 & 1.33 & 0.04 & 2 UCSB & 4.92 & 100.46 \\
\hline 11 & 3696 & -12.00 & -94.00 & 3664 & 2747.1 & $\begin{array}{l}1.78 \\
1\end{array}$ & 34.68 & 27.73 & 145.9 & 138.6 & 36.15 & 2.62 & 3.83 & 1.24 & 0.14 & 2 UCSB & 4.93 & 102.45 \\
\hline 11 & 3695 & -12.00 & -94.00 & 3664 & 2996.2 & $\begin{array}{l}2.78 \\
\end{array}$ & 34.68 & 27.73 & 144 & 141.73 & 36.35 & 2.64 & 3.90 & 1.29 & 0.08 & 2 UCSB & 4.95 & 105.38 \\
\hline 11 & 3692 & -12.00 & -94.00 & 3664 & 3495.8 & \begin{tabular}{l|l}
3 & 1.80
\end{tabular} & 34.69 & 27.74 & 142.5 & 142.21 & 36.44 & 64 & 3.90 & 1.29 & 0.20 & 2 UCSB & 4.96 & 105.77 \\
\hline 11 & 3690 & -12.00 & -94.00 & 3664 & 3620.5 & \begin{tabular}{|l|l|}
5 & 1.81 \\
\end{tabular} & 34.69 & 27.74 & 2.2 & 142.41 & & 54 & 3.91 & 1.28 & 0.14 & 5 UCSB & 4.96 & 106 \\
\hline 11 & 3689 & -12.00 & -94.00 & 3664 & 3639.3 & \begin{tabular}{|l|l|}
3 & 1.81 \\
\end{tabular} & 34.69 & 27.74 & 142.2 & 142.7 & 36.49 & 2.64 & 3.91 & $1.69 \sim$ & & UCSB & 4.96 & 106.21 \\
\hline 15 & 8163 & -16.00 & -104.00 & 3937 & 50.4 & 22.33 & 35.96 & 24.85 & 218.8 & 1.17 & 0.84 & 0.25 & 1.39 & 3.41 & 0.02 & 2 GEOMAR & 0.16 & 0.33 \\
\hline 15 & 8161 & -16.00 & -104.00 & 3937 & 104.6 & 21.76 & 35.99 & 25.04 & 216.9 & 1.37 & 0.96 & 0.29 & 1.43 & 3.31 & 0.19 & 2 GEOMAR & 0.31 & 0.41 \\
\hline 15 & 8159 & -16.00 & -104.00 & 3937 & 183.9 & 18.40 & 35.37 & 25.46 & 192.7 & 46 & 4.93 & 0.54 & 0.30 & 2.98 & 0.18 & 3 GEOMAR & 0.38 & -3.47 \\
\hline 15 & & -16.00 & & & 275.2 & 212.20 & & & & 12.1 & 20.04 & 1.8 & 0.60 & 1.86 & 0.24 & 4 GEOMAR & 2.49 & -7.94 \\
\hline 15 & 8154 & -16.00 & -104.00 & 3937 & 408.8 & 8.73 & 34.58 & 26.84 & 23.3 & 29.48 & 36.85 & 2.66 & 0.80 & 1.58 & $0.143 /$ & 3/1 GEOMAR/UCSB & 3.38 & -7.37 \\
\hline 15 & 3961 & -16.00 & -104.00 & 3933 & 1249.6 & $\begin{array}{l}5.57 \\
\end{array}$ & 34.56 & 27.48 & 82.4 & 98.49 & 41.26 & 3 & 2.39 & 1.39 & $0.142 /$ & 2/2 GEOMAR/UCSB & 4.59 & 57.23 \\
\hline 15 & & -16.00 & -104.00 & 3933 & 1747.5 & 2.57 & 34.62 & 27.62 & 108.5 & 122.89 & 39.05 & 2.86 & 3.15 & 1.38 & 0.16 & 4 UCSB & 4.81 & 83.84 \\
\hline 15 & & -16.00 & & & 2198.9 & 2.04 & 34.65 & & & & & 2.7 & 3.52 & 1.28 & 0.20 & 4 UCSB & .87 & 93.17 \\
\hline 15 & 3955 & -16.00 & -104.00 & 3933 & 2398.3 & 1.90 & 34.67 & 27.71 & 144.3 & 132.06 & 36.21 & 2.65 & 3.65 & $\begin{array}{l}1.36 \\
1.32\end{array}$ & 0.03 & 2 UCSB & 4.88 & 95.85 \\
\hline 15 & 8211 & -16.00 & -104.00 & 3938 & 2795.4 & 1.82 & 34.68 & 27.73 & 145.3 & 137.14 & 36.14 & 2.62 & 3.79 & 1.29 & 0.22 & 2 UCSB & 4.92 & 101 \\
\hline 15 & & -16.00 & -104.00 & 3938 & 3246.6 & 1.81 & 34.68 & 27.73 & & 144.36 & 36.6 & .65 & 3.94 & 1.35 & 0.32 & 4 UCSB & 4.97 & 107.76 \\
\hline 15 & & -16.00 & & 3938 & 3744.8 & 1.83 & 34.68 & 27.73 & 7.6 & 149.63 & 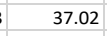 & 88 & 4 & 1.33 & 0.23 & UCSB & 01 & 112.61 \\
\hline 15 & 8204 & -16.00 & -104.00 & 3938 & 3894.8 & 1.84 & 34.68 & 27.73 & 7.2 & 150.22 & 36.98 & 2.68 & 4.06 & 1.32 & 0.19 & 2 UCSB & 5.01 & 113.24 \\
\hline 15 & 8203 & -16.00 & -104.00 & 3938 & 3919.4 & $\begin{array}{l}1.85 \\
\end{array}$ & 34.68 & 27.73 & 77.6 & 150.9 & 36.99 & 2.69 & 4.08 & 1.29 & 0.31 & 3 UCSB & 5.02 & 113.91 \\
\hline 18 & & $\begin{array}{l}-14.98 \\
\end{array}$ & -112.75 & 2644 & \begin{tabular}{|l|l|}
19.7 \\
\end{tabular} & 24.31 & 35.84 & 24.19 & 2.1 & 1.56 & 3.79 & 0.42 & 0.41 & 3.09 & 0.24 & 2 GEOMAR & 44 & -2.23 \\
\hline 18 & 1 & $\begin{array}{l}-14.98 \\
\end{array}$ & -1 & 14 & 95.4 & 22.49 & 36.18 & 24.98 & 209.8 & 17 & 0.29 & 0.3 & 03 & 3.51 & 0.07 & 2 GEOI & 16 & 0.88 \\
\hline 18 & 8449 & -14 & -1 & 2644 & 201.8 & 18.86 & 35.53 & 25.46 & 193.9 & 1.07 & 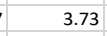 & 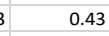 & 0.29 & 3.16 & 0.14 & 2 GEON & 0.07 & -2.66 \\
\hline 18 & 8448 & -14 & -11 & 2644 & 303.4 & 12.00 & 34.62 & 26. & 5 & 16.89 & 23 & 2.19 & 0.73 & 1.58 & $0.393 /$ & $3 / 1$ GEOM & 2.83 & -6.18 \\
\hline 18 & 8446 & -14.98 & -112 & 2644 & 396.7 & 9.16 & 34.61 & 26.78 & $2 \varepsilon$ & 27.23 & & 2.55 & 0.79 & 1.65 & $0.342 /$ & $2 / 1$ GEOM & 3.30 & -7.4 \\
\hline 18 & 8444 & -14.98 & -11 & 2644 & 795.4 & 5.23 & 34.50 & 27.25 & 5 & 58.86 & 40.81 & 2.8 & 1.44 & 1.69 & $0.291 /$ & $1 / 4$ GEOMA & .08 & 18.05 \\
\hline 18 & & -14. & -1 & 2643 & 1193.5 & 3.61 & 34.56 & & & & & .88 & 2.35 & 1.38 & $0.132 /$ & $2 / 3$ GEOMA & 57 & 5.32 \\
\hline 18 & 85 & -14.98 & -112.75 & 2643 & 2186.1 & 1.95 & 34.66 & 27.70 & 141.1 & 129.14 & & 2.6 & 3.54 & $1.23 \sim$ & & 1 UCSB & 86 & 92.67 \\
\hline 18 & 8504 & -14.98 & -112.75 & 2643 & 2211.3 & 1.93 & 34.66 & 27.71 & 143.8 & 128.45 & 36.18 & 2.59 & 3.55 & 1.56 & 0.08 & $2 \mathrm{UC}$ & 86 & 92.27 \\
\hline 18 & 8500 & -14.98 & -112.75 & 2643 & 2311.7 & 1.87 & 34.67 & 27.71 & 152.2 & 126.79 & 35.54 & .56 & 3.57 & 1.38 & 0.01 & 2 UCSB & 84 & 91.25 \\
\hline 18 & 8624 & -14.98 & -112.75 & 2644 & 2346.2 & 1.87 & 34.67 & 27.72 & 152.5 & .38 & 33 & 52 & 3.61 & 1.31 & 0.07 & 3 UCSB & .85 & 92.05 \\
\hline 18 & & -14 & -112 & 2644 & 2421.6 & 18 & & 27 & & & 35 & & 8 & 1.30 & 011 & 5 UCSB & 87 & 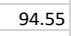 \\
\hline 18 & 86 & -14 & -112.75 & 2644 & 2469.8 & 1.84 & 34.67 & 27.72 & 151.7 & 71 & 35.3 & 2.5 & 3.73 & 1.36 & 0.18 & 2 UCSB & 4.88 & 96.47 \\
\hline 18 & 8617 & -14.98 & -112.75 & 2644 & 2521 & 1.84 & 34.68 & 27.72 & 151.6 & 132.26 & 35.37 & 56 & 3.74 & 1.26 & 0.08 & 2 UCSB & 4.88 & 96.89 \\
\hline 18 & 8613 & -14.98 & -112.75 & 2644 & 2620 & 1.83 & 34.68 & 27.72 & 153.6 & 131.67 & 35.22 & 2.57 & 3.74 & 1.34 & 0.07 & 3 UCSB & 4.88 & 96.45 \\
\hline
\end{tabular}


Table S1. Continued

\begin{tabular}{|c|c|c|c|c|c|c|c|c|c|c|c|c|c|c|c|c|c|c|}
\hline Station & $\begin{array}{l}\text { GEOTRACES } \\
\text { SAMPLE Nr. }\end{array}$ & Lat & Long & $\begin{array}{c}\text { Bottom Depth } \\
(\mathrm{m})\end{array}$ & $\begin{array}{c}\text { DEPTH } \\
(\mathrm{m})\end{array}$ & $\begin{array}{c}\text { Temp } \\
\left({ }^{\circ} \mathrm{C}\right)\end{array}$ & Salinity & $\begin{array}{c}\text { Pot. } \\
\text { Density } \\
\text { (kg/m3) }\end{array}$ & $\left.\begin{array}{c}\text { Oxygen } \\
(\mu \mathrm{mol} / \mathrm{kg})\end{array}\right)$ & $\begin{array}{c}\text { DSi } \\
(\mu \mathrm{mol} / \mathrm{kg})\end{array}$ & $\begin{array}{c}\text { Nitrate } \\
\text { (umol/kg) }\end{array}$ & $\begin{array}{l}\text { Phosphate } \\
\text { (umol/kg) }\end{array}$ & $\begin{array}{c}\text { Dsi/N } \\
\text { (umol/kg) }\end{array}$ & $\begin{array}{l}\delta^{30} \mathbf{S i} \\
(\% \circ)\end{array}$ & 2 s.d. & Lab & $\ln (D s i)$ & $\mathbf{s i}^{*}$ \\
\hline 23 & 9057 & -14.00 & -120.00 & 3404 & 50.1 & 24.75 & 36.15 & 24.28 & 212.5 & 1.85 & 1.17 & 0.3 & 1.58 & 3.50 & 0.20 & 1 GEOMAR & 0.62 & 0.68 \\
\hline 23 & 9052 & -14.00 & -120.00 & 3404 & 227.6 & 17.27 & 35.26 & 25.65 & 183.1 & 1.85 & 6.75 & 0.66 & 0.27 & 2.93 & 0.25 & 4 GEOMAR & 0.62 & -4.9 \\
\hline 23 & 9051 & -14.00 & -120.00 & 3404 & 289.7 & 12.75 & 34.67 & 26.19 & 109.4 & 9.57 & 18.17 & 1.63 & 0.53 & & & UCSB & 2.26 & -8.6 \\
\hline 23 & 9050 & -14.00 & -120.00 & 3404 & 347.8 & 10.73 & 34.63 & 26.53 & 28.9 & 22.55 & 27.49 & 2.47 & 0.82 & 1.75 & 0.363 & 3/1 GEOMAR/UCSB & 3.12 & -4.94 \\
\hline 23 & 9048 & -14.00 & -120.00 & 04 & 745.8 & 5.51 & 34.50 & 27.22 & 109.6 & .23 & 51 & 51 & 1.43 & 1.43 & 0.30 & & 96 & 15.72 \\
\hline 23 & 9047 & -14.00 & -120.00 & 24 & 994.7 & 4.36 & 34.52 & 27.37 & 113.9 & 82 & 58 & 63 & 1.94 & 1.56 & & 1 UCSB & 29 & 35.24 \\
\hline 23 & 8976 & -14.00 & -120.00 & 03 & 992.7 & 4.33 & 34.52 & 27.37 & 116.3 & 3.6 & 37.39 & 61 & 1.97 & 1.52 & & 1 UCSB & 4.30 & 36.21 \\
\hline 23 & 8975 & -14.00 & -120.00 & 3403 & 1242.9 & 3.62 & 34.55 & 27.47 & 116.2 & 90.39 & 38.02 & 69 & 2.38 & 1.50 & 0.182 & 12 GEOMAR/UCSB & 4.50 & 52.37 \\
\hline 23 & 8974 & $\begin{array}{l}-14.00 \\
-14.00\end{array}$ & $\begin{array}{l}-120.00 \\
-120.00\end{array}$ & $\begin{array}{l}3403 \\
3403\end{array}$ & $\begin{array}{r}1489.9 \\
2087\end{array}$ & $\begin{array}{l}2.99 \\
2.04\end{array}$ & $\begin{array}{l}34.58 \\
34.65\end{array}$ & $\begin{array}{l}27.55 \\
27.69\end{array}$ & $\begin{array}{ll}120.6 \\
143.7\end{array}$ & $\begin{array}{l}105.91 \\
125.82\end{array}$ & $\begin{array}{r}37.9 \\
36.19\end{array}$ & .72 & 2.79 & 1.37 & 0.03 & 2 UCSB & 4.66 & 68.01 \\
\hline 23 & $\begin{array}{l}8971 \\
8970\end{array}$ & $\begin{array}{l}-14.00 \\
-14.00\end{array}$ & $\begin{array}{l}-120.00 \\
-120.00\end{array}$ & $\begin{array}{l}3403 \\
3403\end{array}$ & $\begin{array}{r}2087 \\
2186.4\end{array}$ & $\begin{array}{l}2.04 \\
1.94\end{array}$ & $\begin{array}{l}34.65 \\
34.66\end{array}$ & $\begin{array}{l}27.69 \\
27.70\end{array}$ & $\begin{array}{l}143.7 \\
148.7\end{array}$ & $\begin{array}{l}125.82 \\
127.18\end{array}$ & $\begin{array}{l}36.19 \\
35.79\end{array}$ & $\begin{array}{l}2.61 \\
2.59\end{array}$ & $\begin{array}{l}3.48 \\
3.55\end{array}$ & $\begin{array}{l}1.49 \\
1.34\end{array}$ & $\begin{array}{l}0.14 \\
0.30\end{array}$ & $\begin{array}{l}3 \text { UCSB } \\
2 \text { USB }\end{array}$ & $\begin{array}{l}4.83 \\
4.85 \\
4\end{array}$ & $\begin{array}{l}89.63 \\
91.39\end{array}$ \\
\hline 23 & 8968 & -14.00 & -120.00 & 3403 & $\begin{array}{l}2800.4 \\
2335.7\end{array}$ & $\begin{array}{l}1.94 \\
1.88\end{array}$ & $\begin{array}{l}34.66 \\
34.67\end{array}$ & $\begin{array}{l}27.70 \\
27.71\end{array}$ & $\begin{array}{l}148.7 \\
150.5\end{array}$ & $\begin{array}{l}127.18 \\
129.92\end{array}$ & $\begin{array}{l}35.79 \\
35.61\end{array}$ & $\begin{array}{l}2.59 \\
2.58\end{array}$ & $\begin{array}{l}3.55 \\
3.65\end{array}$ & $\begin{array}{l}1.34 \\
1.35\end{array}$ & $\begin{array}{l}0.30 \\
0.08\end{array}$ & $\begin{array}{l}2 \text { UCSB } \\
2 \text { UCSB }\end{array}$ & $\begin{array}{l}4.85 \\
4.87\end{array}$ & $\begin{array}{l}91.39 \\
94.31\end{array}$ \\
\hline 23 & 8965 & -14.00 & -120.00 & 3403 & 2484.6 & 1.83 & 34.67 & 27.72 & 151.4 & 132.36 & 35.62 & 2.58 & $\begin{array}{l}3.65 \\
3.72\end{array}$ & $\begin{array}{l}1.35 \\
1.44\end{array}$ & $\begin{array}{l}0.08 \\
0.23\end{array}$ & 2 UCSB & $\begin{array}{l}4.81 \\
4.89\end{array}$ & $\begin{array}{l}94.31 \\
96.74\end{array}$ \\
\hline 23 & 9107 & -14.00 & -120.00 & 3400 & 2583.2 & 1.81 & 34.67 & 27.72 & 152.3 & 133.23 & 35.78 & 2.59 & 3.72 & $\begin{array}{l}1.44 \\
1.22\end{array}$ & 0.11 & UCSB & $\begin{array}{l}4.89 \\
4.89\end{array}$ & $\begin{array}{l}90.44 \\
97.45\end{array}$ \\
\hline 23 & 9104 & -14.00 & -120.00 & 3400 & 2882 & 1.70 & 34.68 & 27.74 & 158.6 & 4.01 & 35.51 & .58 & 3.77 & 1.24 & 0.00 & UCSB & 4.90 & 98.5 \\
\hline & & & & & & & & & & & & 58 & 3.81 & & 0.31 & UCSB & 4.90 & $\begin{aligned} 98.5 \\
99.47\end{aligned}$ \\
\hline 28 & 9646 & -11.63 & -132.50 & 4041 & & 27.28 & 36.01 & 23.39 & 201.5 & 1.66 & 2.52 & 0.36 & 6.66 & 3.42 & 0.11 & $G E$ & 0.51 & -0.86 \\
\hline 28 & 9644 & -11.63 & -132.50 & 4041 & 59 & 27.23 & 36.01 & 23.41 & 201.9 & 1.6 & 2.46 & 0.37 & 0.67 & 3.25 & 0.11 & GEOMAR & .51 & $\begin{array}{l}-0.00 \\
-0.8 \\
-10\end{array}$ \\
\hline 28 & 9640 & -11.63 & -132.50 & 4041 & 218.6 & 18.01 & 35.41 & 25.58 & 166.7 & 2.34 & 8.19 & 0.8 & 0.29 & 2.45 & 0.20 & 2 GEOMAR & 0.85 & -5.85 \\
\hline 28 & 9639 & -11.63 & -132.50 & 4041 & 267.6 & 14.04 & 34.91 & 26.11 & 96.7 & 9.27 & 18.01 & 1.61 & 0.51 & 1.72 & 0.073 & 3 GEOMAR/UCSB & 2.23 & -8.74 \\
\hline 28 & 9638 & -11.63 & -132.50 & 4041 & 338.1 & 10.56 & 34.70 & 26.62 & 38.7 & 24.21 & 30.33 & 2.37 & 0.80 & 1.67 & 0.18 & 2 GEOMAR & 19 & -6.12 \\
\hline 28 & 9636 & -11.63 & -132.50 & 4041 & 608 & 6.95 & 34.57 & 27.09 & 54.9 & .29 & 39.75 & 2.71 & 1.14 & 1.53 & 0.15 & 2 UCSB & 3.81 & 5.54 \\
\hline 28 & 9635 & -11.63 & -132.50 & 4041 & 857.2 & 5.29 & 34.53 & 27.27 & 65.9 & 1.62 & 41.75 & 2.89 & 1.55 & 1.55 & & 1 UCSB & 4.17 & 22.87 \\
\hline 28 & & -11.63 & -132.50 & 4027 & 852.7 & 5.29 & 34.52 & 27.26 & 66.7 & .55 & 41.4 & 88 & 1.54 & 1.47 & 0.03 & 2 UCSB & 15 & 22.15 \\
\hline 28 & & -11.63 & $\begin{array}{r}-132.50 \\
12.50\end{array}$ & 4027 & 1190.3 & 3.69 & 34.56 & 27.47 & & 4.1 & 38.87 & 88 & 2.42 & 1.45 & 0.132 & $2 / 1 \mathrm{G}$ & 54 & 55.23 \\
\hline 28 & & -11.63 & -132.50 & 4027 & 1738.2 & 2.52 & 34.62 & 27.62 & & & & & .18 & 1.45 & 0.41 & & 78 & 81.36 \\
\hline $\begin{array}{l}28 \\
28\end{array}$ & 9578 & $\begin{array}{l}-11.63 \\
-11.63\end{array}$ & $\begin{array}{l}-132.50 \\
-13250\end{array}$ & 4027 & 2087 & 2.04 & 34.65 & 27.69 & 138.7 & 9.82 & 36.5 & 54 & 3.56 & 1.32 & 0.37 & 20 & 87 & 93.32 \\
\hline $\begin{array}{l}28 \\
28\end{array}$ & $\begin{array}{l}9576 \\
9574\end{array}$ & $\begin{array}{l}-11.63 \\
-11.63\end{array}$ & $\begin{array}{l}-132.50 \\
-132.50\end{array}$ & $\begin{array}{l}4027 \\
4027\end{array}$ & $\begin{array}{l}2284.3 \\
2435.2\end{array}$ & $\begin{array}{l}1.91 \\
1.85\end{array}$ & $\begin{array}{l}34.66 \\
34.67\end{array}$ & $\begin{array}{l}27.71 \\
27.72\end{array}$ & $\begin{array}{r}143.1 \\
145\end{array}$ & $\begin{array}{l}3.33 \\
.19\end{array}$ & $\begin{array}{l}36.13 \\
35.91\end{array}$ & $\begin{array}{r}2.62 \\
2.6\end{array}$ & $\begin{array}{l}3.69 \\
3.76\end{array}$ & 1.39 & $\sim^{0.07}$ & $\begin{array}{l}2 \text { UCSB } \\
1 \text { USB }\end{array}$ & 4.89 & 97.2 \\
\hline 28 & 9657 & -11.63 & $\begin{array}{l}-132.50 \\
-132.50\end{array}$ & 4038 & $\begin{array}{r}2435.2 \\
2981\end{array}$ & $\begin{array}{l}1.85 \\
1.68\end{array}$ & $\begin{array}{l}34.67 \\
34.68\end{array}$ & $\begin{array}{l}27.72 \\
27.74\end{array}$ & $\begin{array}{r}145 \\
156.3\end{array}$ & 7.19 & $\begin{array}{l}35.91 \\
35.59\end{array}$ & $\begin{array}{r}2.6 \\
2.56\end{array}$ & $\begin{array}{l}3.76 \\
3.86\end{array}$ & $\begin{array}{l}1.31 \\
1.29\end{array}$ & 0.05 & $\begin{array}{l}1 \text { UCSB } \\
2 \text { UCSB }\end{array}$ & $\begin{array}{l}4.91 \\
4.92\end{array}$ & $\begin{array}{r}99.28 \\
101.94\end{array}$ \\
\hline 28 & & -11.63 & -132 & & 3229 & 1.60 & 34.69 & 27.75 & 161.4 & & & 4 & 3.90 & 1.32 & $\begin{array}{l}0.08 \\
0.08\end{array}$ & & $\begin{array}{l}4.92 \\
4.92 \\
-9\end{array}$ & $\begin{array}{l}101.94 \\
102.09\end{array}$ \\
\hline 28 & 9655 & -11.63 & -132.50 & 4038 & 3478.2 & 1.57 & 34.69 & 27.76 & 164.2 & .04 & & 52 & 3.91 & 1.42 & & 1 UCSB & $\begin{array}{l}4.92 \\
4.92\end{array}$ & 102.09 \\
\hline 28 & & -11.63 & -132.50 & 4038 & 3875 & 1.56 & 34.69 & 27.76 & 166.9 & 137.14 & 34.92 & & 3.93 & 1.38 & & & & $\begin{array}{l}101.96 \\
102.22\end{array}$ \\
\hline 28 & & & & & 3989.3 & & & & & & & & 3.91 & 1.33 & 0.15 & & 2 & $\begin{array}{l}102.22 \\
101.91\end{array}$ \\
\hline 32 & & -11. & & & 30 & 28.26 & & & & & $\frac{2 .}{2 .}$ & & & 3.32 & & & & -0.67 \\
\hline 32 & & -11 & & & & 27. & & & & & & & & 3.28 & 0.26 & & & $\begin{array}{l}-0.07 \\
-0.65\end{array}$ \\
\hline 32 & & -11 & -14 & 4956 & 124.7 & 26.76 & 35.95 & 23 & 6 & 2 & 18 & 0.53 & 0.64 & 2.77 & 0.07 & 2 GEOM & 81 & -1.24 \\
\hline 32 & & -11 & -142 & & & 20.41 & & & & & 7.01 & 0.7 & 0.31 & 2.71 & 0.24 & 3 GEOM & 77 & -4.86 \\
\hline 32 & & -11 & -142 & & 334.7 & 10.68 & 34.66 & & & & 27.56 & & 4 & 1.48 & 0.163 & 3 GEOMA & 01 & $\begin{array}{l}-7.00 \\
-7.26 \\
\end{array}$ \\
\hline 32 & & -11 & -142 & & & 8.66 & & 26.89 & 103.2 & & 30.33 & & 0.93 & 1.40 & 0.062 & MAR/ & 3.34 & -2.02 \\
\hline 32 & & -11. & -142 & & & 5.25 & & & 78.4 & & & & 1.61 & 1.26 & & & & .33 \\
\hline 32 & & -11 & & & & & & & 8 & & & & & 1.34 & 0.24 & 24 & & \\
\hline 32 & & & & & 12 & 3 & & & & & & & & 1.41 & 0.032 & $12 \mathrm{G}$ & 54 & \\
\hline 32 & & -11 & -142 & & 1492.4 & 3.01 & & & & & & & 8 & 1.36 & 0.10 & & 59 & 86 \\
\hline 32 & & -11 & & & & 2.51 & & & & & & & & 1.34 & 0.22 & & 79 & 98 \\
\hline 32 & gc & $\begin{array}{l}-11.0 \\
-11.0 \\
\end{array}$ & $\begin{array}{r}-142.95 \\
-142.95\end{array}$ & 4954 & $\begin{array}{r}2087 \\
23855\end{array}$ & 2.07 & $\begin{array}{l}34.65 \\
34.67\end{array}$ & 27.71 & 137.6 & & & & 8 & 1.32 & 0.18 & & 87 & 1.2 \\
\hline $\begin{array}{l}32 \\
32 \\
22\end{array}$ & $\begin{array}{l}9956 \\
9953\end{array}$ & -11.0 & $\begin{array}{r}-142.95 \\
-142.95\end{array}$ & $\begin{array}{l}4954 \\
4954\end{array}$ & $\begin{array}{r}2385.5 \\
2585.2\end{array}$ & 1.89 & $\begin{array}{l}34.67 \\
34.67\end{array}$ & 27.71 & $\begin{array}{r}142 \\
1453\end{array}$ & 137.14 & 36.26 & $\begin{array}{r}2.6 \\
2.59\end{array}$ & $\begin{array}{l}3.78 \\
3.85\end{array}$ & 1.26 & 0.23 & 4 UCSB & .92 & 100.88 \\
\hline 32 & & $\begin{array}{l}-11 \\
-11 \\
-11\end{array}$ & $\begin{array}{l}-14 \\
-14\end{array}$ & & 25 & $\begin{array}{l}1.81 \\
1.76\end{array}$ & & & 148.5 & & & & 3.90 & $\begin{array}{l}1.37 \\
1.26\end{array}$ & $\begin{array}{l}0.16 \\
0.15\end{array}$ & & & 56 \\
\hline 32 & & & & & 3477.8 & 1.55 & & & 1 & & & & 7 & 1.32 & 0.22 & & 3 & 103.65 \\
\hline 32 & & -11 & & 4 & 30 & 1.42 & & & & & 33.98 & 2.44 & 4 & 1.32 & 0.01 & 24 & oo & 99.74 \\
\hline 32 & & & & & 44 & 1.38 & & & & & & 2 & & 1.39 & 0.03 & & 38 & 97 \\
\hline 32 & & -11.03 & & & 4719.9 & 1.39 & & 27.78 & & & & & (2) & 1.47 & 0.07 & 2 UCSB & .88 & 7.7 \\
\hline 32 & 10067 & -11.03 & -142.95 & 4954 & 4901.8 & 1.41 & 34.70 & 27.78 & 185.5 & 132.16 & 33.47 & 2.4 & 3.95 & 1.37 & 0.08 & 3 UCSB & 4.88 & 98.69 \\
\hline
\end{tabular}


Table S1. Continued

\begin{tabular}{|c|c|c|c|c|c|c|c|c|c|c|c|c|c|c|c|c|c|c|}
\hline Station & $\begin{array}{l}\text { GEOTRACES } \\
\text { SAMPLE Nr. }\end{array}$ & Lat & Long & $\begin{array}{l}\text { Bottom Depth } \\
\text { (m) }\end{array}$ & $\begin{array}{c}\text { DEPTH } \\
(\mathrm{m})\end{array}$ & $\begin{array}{l}\text { Temp } \\
\left({ }^{\circ} \mathrm{C}\right)\end{array}$ & Salinity & $\begin{array}{c}\text { Pot. } \\
\text { Density } \\
\text { (kg/m3) }\end{array}$ & $\begin{array}{c}\begin{array}{c}\text { Oxygen } \\
(\mu \mathrm{mol} / \mathrm{kg})\end{array}\end{array}$ & $\begin{array}{c}\mathrm{DSi} \\
(\mu \mathrm{mol} / \mathrm{kg})\end{array}$ & $\begin{array}{c}\text { Nitrate } \\
\text { (umol/kg) }\end{array}$ & $\begin{array}{l}\text { Phosphate } \\
\text { (umol/kg) }\end{array}$ & $\begin{array}{c}\begin{array}{c}D s i / N \\
\text { (umol/kg) }\end{array}\end{array}$ & $\begin{array}{l}\delta^{30} \mathbf{S i} \\
(\%)\end{array}$ & 2 s.d. & Lab & $\ln$ (Dsi) & $\mathrm{Si}^{*}$ \\
\hline 36 & 10302 & -10.50 & -152.00 & 5136 & 128.6 & 25.34 & 36.20 & 24.15 & 178.6 & 1.66 & 1.27 & 0.37 & 1.31 & 2.61 & 0.04 & 2 GEOMAR & 0.51 & 0.39 \\
\hline 36 & 10300 & -10.50 & -152.00 & 5136 & 298.7 & 14.34 & 34.99 & 26.11 & 104.2 & 9.27 & 17.6 & 1.49 & 0.53 & 1.80 & 0.06 & 2 GEOMAR/UCSB & 2.23 & -8.33 \\
\hline 36 & 10299 & -10.50 & -152.00 & 5136 & 348.5 & 10.75 & 34.71 & 26.59 & 75.7 & 20.11 & 26.98 & 2.07 & 0.75 & 1.63 & 0.16 & 2/1 GEOMAR/UCSB & 3.00 & -6.87 \\
\hline 36 & 10297 & -10.50 & -152.00 & 5136 & 596.7 & 6.79 & 34.55 & 27.09 & 95.7 & 42.76 & 35.44 & 2.45 & 1.21 & 1.55 & 0.23 & 2/1 GEOMAR/UCSB & 3.76 & 7.32 \\
\hline 36 & 10295 & -10.50 & -152.00 & 5136 & 995.5 & 4.47 & 34.52 & 27.36 & 112 & 74.97 & 37.61 & 2.66 & 1.99 & 1.41 & & 1 UCSB & 4.32 & 37.36 \\
\hline 36 & 10286 & -10.50 & -152.00 & 5141 & 993.8 & 4.63 & 34.52 & 27.34 & 108.4 & 71.94 & 37.71 & 2.64 & 1.91 & 1.45 & & 1 UCSB & 4.28 & 34.23 \\
\hline 36 & 10285 & -10.50 & -152.00 & 5141 & 1243 & 3.71 & 34.55 & 27.46 & 115 & 92.15 & 37.7 & 2.67 & 2.44 & 1.48 & 0.17 & 2/3 GEOMAR/UCSB & 4.52 & 54.45 \\
\hline 36 & 10281 & -10.50 & -152.00 & 5141 & 2087.7 & 2.06 & 34.65 & 27.68 & 141.6 & 129.33 & 36.35 & 2.6 & 3.56 & 1.37 & 0.11 & $2 U C S B$ & 4.86 & 92.98 \\
\hline 36 & 10277 & -10.50 & -152.00 & 5141 & 2485.9 & 1.83 & 34.67 & 27.72 & 148.4 & 135.09 & 36.01 & 2.57 & 3.75 & 1.30 & 0.12 & 2 UCSB & 4.91 & 99.08 \\
\hline 36 & 10453 & -10.50 & -152.00 & 5147 & 2783.6 & 1.76 & 34.67 & 27.73 & 148.4 & 136.55 & 35.71 & 2.56 & 3.82 & 1.26 & 0.19 & 3 UCSB & 4.92 & 100.84 \\
\hline 36 & 10450 & -10.50 & -152.00 & 5147 & \begin{tabular}{|l|l}
3229.7 \\
\end{tabular} & 1.63 & 34.68 & 27.74 & 158.3 & 136.94 & 35.18 & 2.51 & 3.89 & 1.28 & 0.26 & 3 UCSB & 4.92 & 101.76 \\
\hline 36 & 10449 & -10.50 & -152.00 & 5147 & 3478.8 & 1.55 & 34.69 & 27.75 & 165.5 & 135.28 & 34.66 & 2.48 & 3.90 & 1.34 & 0.19 & 3 UCSB & 4.91 & 100.62 \\
\hline 36 & 10448 & -10.50 & -152.00 & 5147 & 3974.9 & 1.41 & 34.69 & 27.77 & 176.8 & 132.45 & 33.9 & 2.42 & 3.91 & 1.32 & 0.19 & 2 UCSB & 4.89 & 98.55 \\
\hline 36 & 10447 & -10.50 & -152.00 & 5147 & 4474.3 & 1.33 & 34.70 & 27.78 & 188.1 & 128.45 & 33.09 & 2.36 & 3.88 & 1.40 & 0.12 & 3 UCSB & 4.86 & 95.36 \\
\hline 36 & 10446 & -10.50 & -152.00 & 5147 & 4718.7 & 1.31 & 34.70 & 27.79 & 192.5 & 126.2 & 32.73 & 2.33 & 3.86 & 1.27 & 0.08 & 3 UCSB & 4.84 & 93.47 \\
\hline 36 & 10445 & -10.50 & -152.00 & 5147 & 4970.2 & 1.31 & 34.71 & 27.79 & 194.5 & & & 31 & 3.88 & 1.42 & 0.19 & 4 UCSB & 4.83 & 93.35 \\
\hline 36 & 10444 & -10.50 & -152.00 & 5147 & 5069.3 & 1.32 & 34.71 & 27.79 & 195 & 124.93 & 32.43 & 2.31 & 3.85 & 1.25 & & 1 UCSB & 4.83 & 92.5 \\
\hline 36 & 10443 & -10.50 & -152.00 & 5147 & 5094.5 & 1.32 & 34.71 & 27.79 & 195.2 & 124.93 & 32.5 & 2.31 & 3.84 & 1.35 & 0.05 & 4 UCSB & 4.83 & 92.43 \\
\hline
\end{tabular}


Tab. S2. Mean $\delta^{30} \mathrm{Si}$ values for samples from both laboratories. The external reproducibility ( 2 s.d.) of $n$ analytical replicates and the $\%$ difference between both laboratories are overall in very good agreement.

\begin{tabular}{|c|c|c|c|c|c|c|c|}
\hline \multirow[b]{2}{*}{$\begin{array}{c}\delta^{30} \mathrm{Si} \\
\text { GEOMAR }\end{array}$} & \multirow[b]{2}{*}{$\begin{array}{c}2 \text { s.d. } \\
\text { GEOMAR }\end{array}$} & \multirow[b]{2}{*}{ n } & \multirow[b]{2}{*}{$\begin{array}{c}\delta^{30} \mathrm{Si} \\
\mathrm{UCSB}\end{array}$} & \multirow[b]{2}{*}{$\begin{array}{c}2 \text { s.d. } \\
\text { UCSB }\end{array}$} & \multirow[b]{2}{*}{ n } & OFFSET & $\%$ diff \\
\hline & & & & & & $\begin{array}{c}\text { GEOMAR- } \\
\text { UCSB }\end{array}$ & $\begin{array}{c}\text { (GEOMAR- } \\
\text { UCSB)/GEO } \\
\text { MAR*100 }\end{array}$ \\
\hline 1.50 & 0.00 & 2 & 1.53 & $\sim$ & 1 & -0.03 & -2.33 \\
\hline 1.60 & 0.08 & 3 & 1.47 & 0.21 & 3 & 0.13 & 8.04 \\
\hline 1.40 & 0.04 & 2 & 1.33 & 0.28 & 2 & 0.07 & 4.99 \\
\hline 1.59 & 0.10 & 2 & 1.47 & 0.08 & 2 & 0.12 & 7.68 \\
\hline 1.73 & 0.24 & 2 & 1.77 & 0.46 & 4 & -0.04 & -2.33 \\
\hline 1.38 & 0.19 & 2 & 1.46 & 0.30 & 5 & -0.08 & -6.04 \\
\hline 1.42 & 0.05 & 2 & 1.52 & 0.18 & 3 & -0.10 & -7.37 \\
\hline 1.45 & 0.24 & 1 & 1.63 & 0.11 & 3 & -0.17 & -11.82 \\
\hline 1.47 & 0.24 & 2 & 1.46 & 0.12 & 3 & 0.00 & 0.24 \\
\hline 1.49 & 0.22 & 1 & 1.52 & $\sim$ & 1 & -0.03 & -2.06 \\
\hline 1.55 & 0.18 & 1 & 1.69 & 0.27 & 2 & -0.14 & -9.30 \\
\hline 1.43 & 0.12 & 2 & 1.42 & $\sim$ & 1 & 0.01 & 0.90 \\
\hline 1.57 & 0.17 & 3 & 1.59 & $\sim$ & 1 & -0.01 & -0.93 \\
\hline 1.40 & 0.20 & 2 & 1.39 & 0.12 & 2 & 0.01 & 0.52 \\
\hline 1.72 & 0.25 & 3 & 1.45 & $\sim$ & 1 & 0.27 & 15.85 \\
\hline 1.49 & 0.20 & 2 & 1.41 & $\sim$ & 1 & 0.08 & 5.29 \\
\hline 1.56 & 0.15 & 1 & 1.72 & 0.28 & 4 & -0.17 & -10.60 \\
\hline 1.35 & 0.15 & 2 & 1.42 & 0.11 & 3 & -0.07 & -4.82 \\
\hline 1.83 & 0.21 & 3 & 1.51 & $\sim$ & 1 & 0.32 & 17.32 \\
\hline 1.43 & 0.11 & 2 & 1.57 & 0.06 & 2 & -0.14 & -9.74 \\
\hline 1.48 & 0.09 & 2 & 1.39 & $\sim$ & 1 & 0.09 & 6.30 \\
\hline 1.42 & 0.05 & 2 & 1.40 & 0.25 & 2 & 0.02 & 1.53 \\
\hline 1.59 & 0.03 & 2 & 1.72 & $\sim$ & 1 & -0.13 & -8.44 \\
\hline 1.50 & 0.24 & 2 & 1.64 & $\sim$ & 1 & -0.14 & -9.01 \\
\hline 1.46 & 0.12 & 2 & 1.50 & 0.22 & 3 & -0.04 & -2.40 \\
\hline
\end{tabular}


Tab. S3. Mean $\delta^{30} \mathrm{Si}$ values for reference materials measured in both laboratories along with the literature values according to Reynolds et al. (2007) and Grasse et al. (2017). The inter-calibrated seawater standards ALOHA 300 and ALOHA 1000 as well as the solid standards (Diatomite, Big Batch) measured at both laboratories are in very good agreement shown by the $\%$ difference between both laboratories $(<2 \%)$, except for the $\mathrm{ALOHA}_{300}$ with a difference of $25 \%$.

\begin{tabular}{|c|c|c|c|c|c|c|c|c|c|c|c|c|c|c|c|}
\hline & & \multicolumn{6}{|c|}{ GEOMAR } & \multicolumn{6}{|c|}{ UCSB } & \multirow[b]{2}{*}{$\begin{array}{c}\text { OFFSET } \\
\text { UCSB-Kiel }\end{array}$} & \multirow[b]{2}{*}{$\begin{array}{c}\% \\
\text { Difference } \\
\text { (UCSB- } \\
\text { Kiel)/USCB }\end{array}$} \\
\hline & $\begin{array}{c}\text { Reference } \\
\text { Mean }\end{array}$ & $\begin{array}{c}\delta^{30} \mathrm{Si} \\
\text { Mean }\end{array}$ & 2 s.d. & $\begin{array}{c}\delta^{29} \mathrm{Si} \\
\text { Mean }\end{array}$ & 2 s.d. & $\begin{array}{c}\delta^{30} \mathbf{S i} \\
\text { Median }\end{array}$ & $\mathbf{n}$ & $\begin{array}{l}\delta^{30} \mathrm{Si} \\
\text { Mean }\end{array}$ & 2 s.d. & $\begin{array}{c}\delta^{29} \mathbf{S i} \\
\text { Mean }\end{array}$ & 2 s.d. & $\begin{array}{c}\delta^{30} \mathbf{S i} \\
\text { Median }\end{array}$ & $\mathbf{n}$ & & \\
\hline ALOHA1000 & 1.24 & 1.27 & 0.15 & 0.68 & 0.13 & 1.27 & 35 & 1.29 & 0.22 & 0.66 & 0.11 & 1.29 & 35 & 0.02 & 1.32 \\
\hline ALOHA300 & 1.68 & 1.80 & 0.22 & 0.91 & 0.19 & 1.76 & 10 & 1.44 & 0.28 & 0.73 & 0.14 & 1.40 & 12 & -0.36 & -25.22 \\
\hline Diatomite & 1.26 & 1.25 & 0.14 & 0.61 & 0.12 & 1.26 & 9 & 1.26 & 0.20 & 0.64 & 0.10 & 1.26 & 25 & 0.01 & 0.86 \\
\hline BB & -10.48 & -10.56 & 0.26 & -5.44 & 0.14 & -10.56 & 12 & -10.50 & 0.21 & -5.36 & 0.11 & -10.50 & 120 & 0.06 & -0.57 \\
\hline
\end{tabular}

